\title{
Estudio del estadio de cambio y estado emocional en jugadores patológicos
}

\author{
llinares Pellicer, M.C.*; Palau Muñoz, C.**; Santos Diez, P.***; \\ Albiach Catalá, C.****; Camacho Ferrer, I. \\ * Psicóloga. Unidad de Conductas Adictivas de la Mancomunitat de Municipis de la Safor. Área de Salud n 11. Gandia, Valencia. \\ * Psicóloga. Unidad de Conductas Adictivas del Área de Salud n 5. Paterna Valencia. Conselleria de Sanidad. Generalitat Valenciana. \\ ** Dr. en Psicología. Unidad de Conductas Adictivas del Área de Salud n 13. Xàtiva. Valencia. Conselleria de Sanidad. Generalitat Valenciana. \\ *** Psicóloga. Unidad de Conductas Adictivas de la Mancomunitat L’Horta Nord. Área de Salud n 4. Massamagrell, Valencia. \\ **** Psicóloga. Unidad de Conductas Adictivas del Área de Salud n 12. Denia. Alicante. Conselleria de Sanidad. Generalitat Valenciana
}

\section{RESUMEN}

Objetivo: estudiar el estadio de cambio y el estado emocional de los jugadores patológicos al inicio de tratamiento, en cinco Unidades de Conductas Adictivas de la Comunidad Valenciana.

Material y Método: estudio multicéntrico de carácter descriptivo. Instrumentos utilizados: SOGS, BDI, STAI y CBA. Muestra constituida por 133 sujetos (14 mujeres y 119 hombres) adictos al juego. El análisis de los datos realizado con el paquete estadístico SPSS.

Resultados: Todos los sujetos de la muestra cumplían criterios de probable jugador patológico (SOGS). La mitad de la muestra se ubica en el estadio de contemplación. Percentil medio 68.38 para ansiedad/estado y 76.17 para ansiedad/rasgo. EI BDI refleja estado emocional disfórico. Las puntuaciones del CBA indican consumo moderado de alcohol. El 63.2\% no precisa tratamiento farmacológico.

Conclusiones: El alto porcentaje de sujetos que presenta consumo de alcohol concomitante con la conducta de juego, exige una intervención especifica para estos sujetos. Los altos niveles de ansiedad y disforia que presentan los sujetos al inicio de tratamiento, parecen relacionados con los estresores propios de la conducta de juego.

Palabras clave: juego patológico, estadio de cambio, depresión, ansiedad, alcohol.

\section{SUMMARY}

Objective: The purpose of this article is to study the stage of change and the emotional state of pathological gamblers at treatment intake within five drug addiction treatment centres (Unidades de Conductas Adictivas) in the Valencia region, Spain.

Material and Method: A descriptive, multi-centre study. Material used: SOGS, BDI, STAI y CBA. Our sample consists of 133 subjects, 14 females and 119 males -all being addicted to gambling. Data was analysed using the Statistical Package for the Social Sciences (SPSS)

Results: All subjects in our sample meet the SOGS criteria for potential pathological gamblers. Half of the subjects are in a stage of contemplation. The anxiety/state mean percentile is 68.38 and it is 76.17 for anxiety/traits. BDI shows a dysphoric emotional estate. CBA scores show moderate alcohol consumption. $63.2 \%$ of them do not need any pharmacological treatments.

Conclusions: The high percentage of subjects who drink alcohol while gambling requires a specific intervention. High levels of anxiety and dysphoria shown by the subjects at treatment intake seem to be related to stress factors common to gambling behaviours.

Key words: pathological gambling, stage of change, depression, anxiety, and alcohol.

De los países de la Comunidad Económica Europea, España y Alemania son los países que ocupan el primer lugar de gasto per cápita en juego (Becoña, 1996). El gran número y diversificación de juegos existentes en España (máquinas recreativas, varias loterías, el cupón de la ONCE, quinielas, bingo, casinos, etc.) representa un gasto anual de los españoles de 4.1 billones de pesetas (Comisión Nacional del Juego, 2000). El juego más popular es el de las máquinas tra- 
gaperras, el volumen de lo jugado en ellas es de 1.733 millones de pesetas en el 2000 (Comisión Nacional del Juego), lo que supone un incremento respecto a 1999 del 8,89\%.

Los juegos que originan más adicción son los que permiten la mayor proximidad espacial y temporal entre la apuesta y el premio, tal como ocurre, en primer término con las máquinas tragaperras y el bingo.

El incremento del juego en España ha sido exponencial desde su legalización en 1977 (Becoña, 1996). Becoña et al., (1995) estiman la tasa de jugadores patológicos en España de 18 o más años en un 1.5 y la de jugadores problema en un 2.5. Estos datos significarían que el número de jugadores patológicos sería de 450.000 y el de jugadores problema de 750.000.

Este trastorno es más frecuente en hombres que en mujeres, pero éstas son mucho más reacias a buscar ayuda terapéutica por la censura social existente. A diferencia de otras conductas adictivas, el juego patológico se distribuye por todas las clases sociales y por todas las edades. No obstante, la edad de acceso a esta adicción ha descendido en los últimos años. De hecho, cada vez son más los adolescentes que acuden a consulta por problemas de juego (Báez y Echeburúa, 1995).

Desde una perspectiva psicopatológica, los trastornos del estado de ánimo (depresión e hipomanía, fundamentalmente) y las conductas adictivas están asociados con frecuencia con el juego patológico. En una muestra de 64 ludópatas de Báez et al. (1994) un 94\% manifestaba algún grado de sintomatología depresiva. Por otra parte, la tasa de prevalencia del consumo abusivo de alcohol u otras drogas entre ludópatas oscila alrededor del 25\% (Báez et al 1994).

A pesar de que el juego patológico ha existido desde hace muchos años, no fue reconocido oficialmente como trastorno hasta 1980, año en el que la Sociedad Americana de Psiquiatría (American Psychiatric Association), en el DSM-III incluyó el juego patológico como uno de los trastornos del control de los impulsos no clasificados en otros apartados junto a la cleptomanía, la piromanía y el trastorno explosivo intermitente. A partir de la American Psychiatric Association (1987) el juego patológico aparece ya claramente como una dependencia sin drogas. La American Psychiatric Association (1994) tiene dos criterios diagnósticos: el primero consta de una lista de diez situaciones de las que se precisan cinco para hacer el diagnóstico, similares a los de la versión anterior pero refundiendo algunos y añadiendo otros como el que hace referencia a que el jugador juega para aliviar estados afectivos disfóricos como la ansiedad o la tristeza. También incorpora en la lista de síntomas los que se refieren a las "mentiras", "los delitos" y "los préstamos" relacionados con el juego. El segundo es un criterio de "cautela" que no de exclusión diagnós- tica absoluta, para cuando los síntomas se presentan dentro de un episodio maníaco.

Las terapias cognitivo-conductuales son en la actualidad, las que están demostrando mayor eficacia en el tratamiento del juego patológico (Arribas y Martínez, 1991; Echeburúa et al., 1994; Echeburúa, Báez y Fernández, 1996).

Los objetivos del presente estudio se centran en la evaluación del estadio de cambio al inicio de tratamiento de los sujetos que demandan atención por ludopatía en diferentes Unidades de Conductas Adictivas de la Comunidad Valenciana, y en estudiar los niveles de ansiedad y depresión en estos sujetos al inicio de tratamiento.

\section{MATERIALY MÉTODO}

En el presente trabajo se llevó a cabo un estudio multicéntrico de carácter descriptivo. Se valoraron características sociodemográficas, de consumo de drogas legales e ilegales, estadio de motivación al cambio y el estado emocional que presentan los sujetos objeto de nuestro estudio.

Los instrumentos utilizados han sido:

- La entrevista clínica.

- La entrevista estructurada de la Historia de Juego

- El Cuestionario de Juego Patológico de South Oaks (SOGS) (South Oaks Foundation, 1992. Validación española de Echeburúa, Báez, Fernández-Montalvo y Páez, 1994), es un cuestionario de 20 ítmes elaborado con arreglo a los criterios del DSM-III-R (American Psychiatric Association (1987). El SOGS tiene una alta correlación con los criterios del DSM-III-R $(r=0.94 ; p<0.001)$. Presenta una buena consistencia interna (coeficiente alfa de Cronbach $=0.07$; $p<0.001)$, así como una alta correlación test-retest $(r=0.71 ; p<0.01)$. El SOGS se ha validado en población española y presenta una fiabilidad test-retest de 0.98 ( $p<0.001)$ y un índice de consistencia interna de 0.94 .

- El Inventario de Depresión de Beck (BDI) (Beck, Rush, Shaw y Emery, 1979), consta de 21 ítems y mide la intensidad de los síntomas depresivos, sobre todo los relacionados con las alteraciones cognitivas. El coeficiente de fiabilidad por el método de las dos mitades es de 0.93, desde la perspectiva de la validez convergente, la correlación con la evaluación clínica de la depresión oscila de 0.62 a 0.66.

- El Cuestionario de Ansiedad de Spielberg (STAI) (Spielberger, Gorsuch y Lusne, 1970) consta de 20 ítems relacionados con ansiedad-rasgo y otros 20 relacionados con ansiedad-estado. La fiabilidad testretest es de 0.81 en asiedad-rasgo y de 0.40 en 
ansiedad-estado. La consistencia interna oscila de 0.83 a 0.92 .

- El Cuestionario Breve de Alcoholismo (Feuerlein, 1976) consta de 22 ítems con una puntuación dicotómica. La validación española de esta prueba ha sido realizada por A. Rodríguez Martos.

El estadio de motivación al cambio fue evaluado, en todos los casos, por el psicólogo/a de la UCA en la primera sesión. Durante la entrevista clínica se intercalaron las siguientes preguntas: ¿juegas en la actualidad?, ¿estás pensando en dejar de jugar en los próximos seis meses?, ¿te planteas dejar de jugar en los próximos treinta días?, ¿Has dejado de jugar al menos una semana durante el año pasado? y ¿cuánto tiempo llevas sin jugar?. Se trata de una adaptación de un sistema de clasificación categorial basado en una serie de preguntas mutuamente excluyentes desarrollado por DiClemente et al. (1991), Prochaska y DiClemente (1992). Los precontempladores contestan que están jugando en la actualidad y no consideran dejar de jugar en los próximos seis meses. Los contempladores juegan en la actualidad pero consideran dejar de jugar en los próximos seis meses. Los preparados para el cambio juegan en la actualidad pero están planeando dejar de jugar en los próximos treinta días y han permanecido sin jugar por un periodo de al menos una semana durante el año anterior. Los jugadores ubicados en actuación son aquellos que no juegan en la actualidad y presentan una abstinencia al juego inferior a seis meses. Por último, los jugadores que presentan una abstinencia al juego superior a seis meses, se ubicarían en mantenimiento.

El psicólogo del Centro, a partir de la puntuación obtenida en el SOGS (este cuestionario está elaborado con arreglo a los criterios del DSM-III y adaptado al DSM-III-R) realizó el diagnóstico. Los autores de la validación española señalan que la sensibilidad y la especificidad son del 100 y el $98 \%$, por lo que se puede utilizar (al menos en población española) como una prueba propiamente diagnóstica, dado que las diferencias de puntuación entre los jugadores patológicos y los sujetos normativos son muy acentuadas.

La selección de la muestra se realizó a partir de todos aquellos sujetos que demandaron tratamiento, durante el periodo comprendido entre febrero de 1999 y mayo de 2001, por problemática relacionada con el juego y tras ser evaluados cumplían criterios para el diagnóstico de jugador patológico. Fueron excluidos los jugadores problema.

La muestra está constituida por 133 sujetos (14 mujeres y 119 hombres) adictos al juego $(82.7 \%$ máquinas tragaperras, $3.8 \%$ bingo, 1.5\% lotería y $12 \%$ dos o más juegos). Ver gráfica 1.

El análisis de los datos se llevó a cabo con el paquete estadístico SPSS (v. 8.0.1.s).

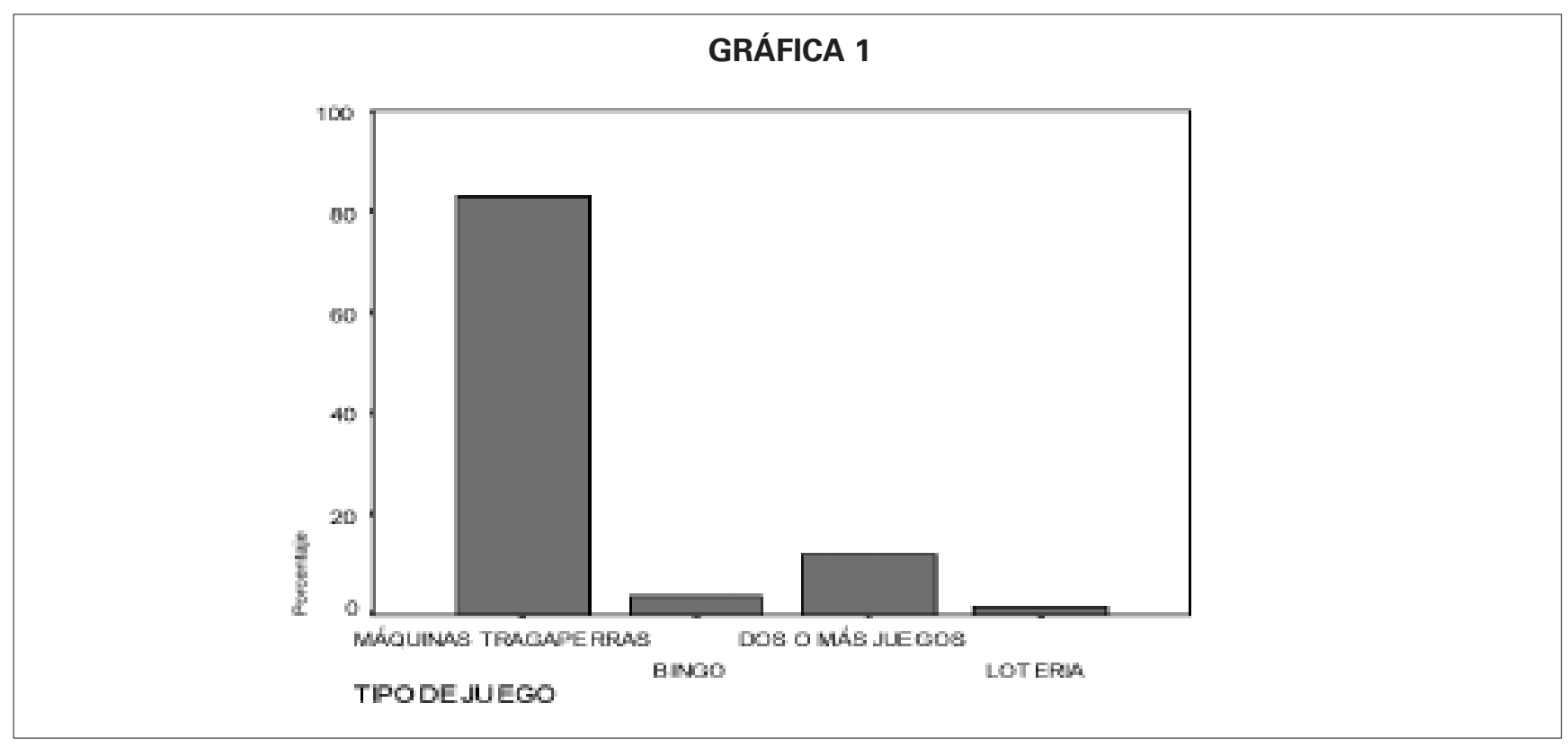

\section{RESULTADOS}

En lo relativo a los datos sociodemográficos obtenemos una edad media de los sujetos de 37 años con una desviación típica de 12.91. En cuanto al estado civil encontramos que el $53.4 \%$ están casado y el
33.3\% solteros. El $75.9 \%$ de la muestra tiene realizada la EGB con o sin graduado escolar. En referencia a la profesión observamos que el $42.1 \%$ son obreros cualificados y el $32.3 \%$ obreros no cualificados. El $69.9 \%$ de la muestra están trabajando mientras que el $10.5 \%$ están parados sin cobrar prestaciones de 
desempleo y otro $10.5 \%$ son pensionistas. En lo relativo al modo de vida, el $54.9 \%$ viven en la familia nuclear frente a un $36.9 \%$ que vive en su familia de origen. Cabe resaltar que el $96.2 \%$ no tiene problemas judiciales.

El 36.8\% realiza la demanda de tratamiento por iniciativa propia, el $21 \%$ por presión familiar y el resto derivado desde otros servicios sociosanitarios (42.2\%). En el momento de acudir a las UCAs el $38.4 \%$ viene acompañado por sus parejas, el $27.1 \%$ acude solo y el resto lo hace acompañado por otros familiares o agentes sociales (trabajadores sociales, educadores, etc.).
En lo referente al tratamiento, un $82 \%$ no ha realizado ningún tratamiento previo, y de los que lo han llevado a cabo el $10.5 \%$ lo han hecho en las Unidades de Salud Mental.

El $88 \%$ de la muestra no presenta antecedentes de alcoholismo familiar, al igual que de patología psiquiátrica. En cuanto a los antecedentes personales, señalar que el $23.3 \%$ presenta psicopatología previa y el $17.3 \%$ antecedentes de alcoholismo.

El $39.8 \%$ de la muestra manifiesta un consumo de alcohol concomitante con la conducta de juego y una pequeña proporción consume drogas ilegales (Ver gráfica 2).

\section{GRÁFICA 2}

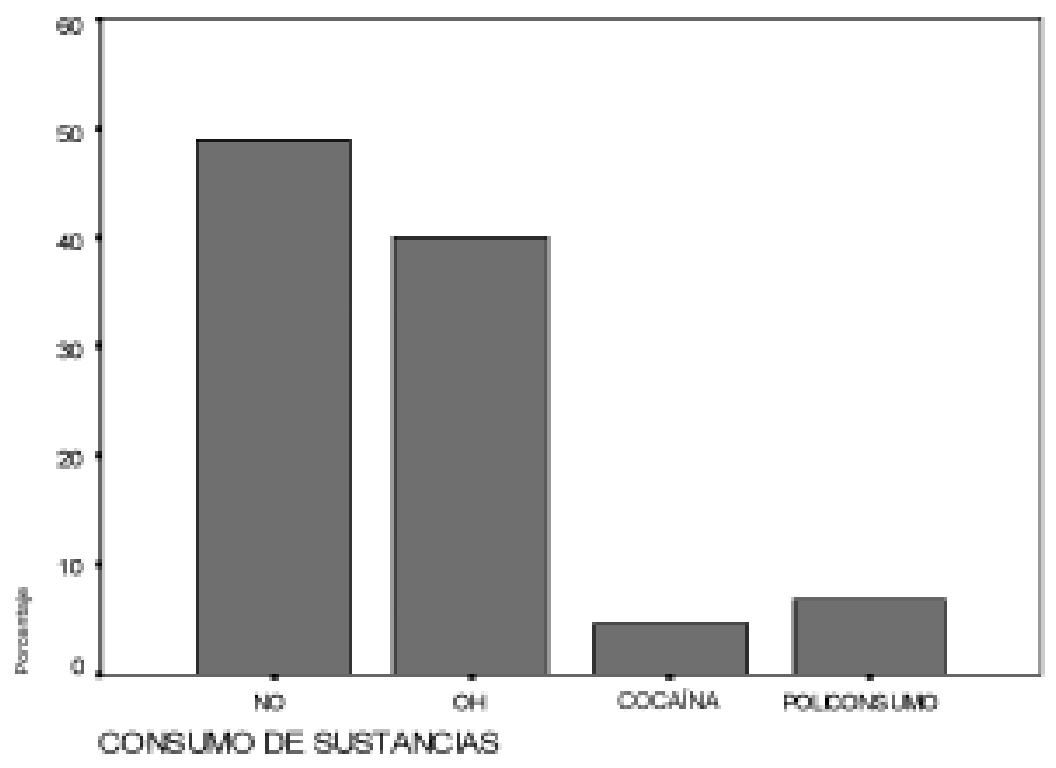

GRÁFICA 2

ESTADIOS DE CAMBIO

actuackon

precomemplacion




TABLA I. Estado emocional

\begin{tabular}{|l|l|c|c|c|}
\hline \multicolumn{2}{|l|}{ P.D.(MEDIA) } & P.C. & D.T. \\
\hline ANSIEDAD & ANSIEDAD/ESTADO & 69.54 & 68.38 & 28.22 \\
& ANSIEDAD/RASGO & 76.44 & 76.17 & 25.10 \\
\hline DEPRESIÓN & BDI & 15.57 & & 10.80 \\
\hline P.D.: Puntuación Directa. & & \\
P.C.: Puntuación Centil. \\
D.T.: Desviación Típica.
\end{tabular}

La mitad de la muestra se ubica en el estadio de contemplación (51.4\%), mientras que el $36.8 \%$ en los estadios de preparación y actuación. Ver gráfica 3.

La evaluación del estado emocional refleja en lo relativo a la ansiedad, una puntuación directa media de 69.54 (percentil 68.38) y para la ansiedad/estado una puntuación directa media de 76.44 (percentil 76.17). La medida proporcionada por el BDI muestra una puntuación media de 15.05 que se correspondería con un estado emocional disfórico. Ver tabla I.

Por último, señalar que la puntuación media obtenida por los sujetos en el CBA indica consumo moderado de alcohol.

El 63.2\% no precisa tratamiento farmacológico. De los que sí lo precisan al $9.8 \%$ se le ha pautado antidepresivos, y al mismo porcentaje ansiolíticos.

\section{DISCUSIÓN}

A partir de los resultados obtenidos observamos características diferenciales respecto al colectivo de adictos a sustancias tanto legales como ilegales: ausencia de antecedentes judiciales, mayor adaptación social, menor presencia de psicopatología asociada y un menor grado de desestructuración familiar.

Más de tres cuartas partes de la muestra no ha realizado tratamientos previos, lo cual nos indicaría que, por un lado, nos encontramos ante una adicción de aparición relativamente nueva; y por otro lado, la creación de las Centros específicos dentro de la asistencia sanitaria pública donde se atienden adicciones tanto químicas como comportamentales, ha facilitado el acceso a tratamiento de dicha patología.

El porcentaje de sujetos que presenta consumo de alcohol concomitante con la conducta de juego, exige una intervención especifica para estos sujetos. En primer lugar, debemos diagnosticar la existencia de abuso o dependencia para, junto con el paciente, decidir el tipo programa a llevar a cabo (motivación, desintoxicación, deshabituación, programa de bebida controlada...).
Consideramos interesante resaltar el bajo porcentaje de sujetos de la muestra usuarios de drogas ilegales. No obstante, en la práctica clínica, observamos pacientes que presentan adicción a sustancias ilegales y que en ocasiones, presentan episodios de juego compulsivo (casi siempre máquinas tragaperras) asociado al consumo de cocaína y alcohol. Somos conscientes de la importancia de realizar una buena anamnesis en sujetos cuya demanda principal es de adicción a sustancias ilegales para poder detectar problemática relacionada con el juego en dichos sujetos.

Las puntuaciones obtenidas con el BDI difieren de las halladas por Becoña (1993), Becoña, Lorenzo y Fuentes (1996), en el sentido de que nuestros pacientes presentan unos niveles de depresión más elevados. Si interpretamos la ausencia de tratamientos previos en nuestra muestra como un mayor tiempo de exposición a la conducta-problema y por tanto, al aumento de problemas asociados, ello redundará en un mayor impacto sobre el estado emocional.

Los altos niveles de ansiedad y disforia que manifiestan los sujetos al inicio de tratamiento, parecen relacionados con los estresores propios de la conducta de juego, es decir, secundarios a la misma.

La mayor parte de los sujetos se encuentran ambivalentes respecto a su decisión de dejar de jugar en el momento en que demandan tratamiento. Por ello, creemos conveniente dedicar las primeras sesiones a trabajar la motivación al cambio. Mediante la entrevista motivacional conseguimos, con mayor probabilidad, que el sujeto supere la ambivalencia propia del estadio de contemplación.

AGRADECIMIENTOS: La presente investigación ha sido becada por la Dirección General de Drogodependencias de la Generalitat Valenciana.

\section{BIBLIOGRAFÍA}

Allcock, C. (1986): Pathological gambling Australian and New Zealand. Journal of psychiatry, 20, 259-265. 
American Psychiatric Association (1980): Diagnostic and statistical manual of mental disorders, 3rd. ed. Washington, D.C.: American Psychiatric Association (trad. cast. en Barcelona: Masson, 1984).

American Psychiatric Association (1987): Diagnostic and statistical manual of mental disorders, revised 3rd. ed. Washington D.C.: American Psychiatric Association (trad. cast. en Barcelona: Masson, 1988).

American Psychiatric Association (1994): Diagnostic and statistical manual of mental disorders: DSM-IV. Washington, D.C.: American Psychiatric Association (trad. cast. en Barcelona: Masson, 1995).

Arribas, M.P. y Martínez, J.J. (1991): Tratamiento individual de jugadores patológicos: descripción de casos. Análisis y modificación de conducta, 17, 255-269.

Báez, C. y Echeburúa, E. (1995): El control de estímulos y la exposición con prevención de respuesta como tratamiento psicológico de la ludopatía en un adolescente. Análisis y modificación de Conducta, 21, 125-145.

Báez, C; Echeburúa, E. y Fernández, J. (1994): Características demográficas, de personalidad y psicopatológicas de los jugadores patológicos de máquinas tragaperras en tratamiento: un estudio descriptivo. Clínica y salud, 5 289-305.

Beck, A.T., Rush, A.J., Shaw, B.F. y Emery, G. (1983): Terapia cognitiva de la depresión. Bilbao. Desclée de Brower (original, 1979).

Becoña Iglesias, E. (1993): El Juego compulsivo en la Comunidad Autónoma Gallega. Santiago de Compostela: Consellería de Sanidad da Xunta de Galicia.

Becoña Iglesias, E. (1995): Tratamiento del juego patológico. En Buceta, J.M. y Bueno, A.M.: Tratamiento psicológico de hábitos y enfermedades. Madrid. Pirámide, pag. 249-278

Becoña Iglesias, E. (1996): Juego patológico: estudios de prevalencia en España. Actas XXIII Jornadas Nacionales de Socidrogalcohol.

Becoña, E.; Lorenzo, M.C. y Fuentes, M.J. (1996): Pathological gambling and depression. Psychological reports, 78,635-640.
Echeburúa, E., Báez, C. (1994): Concepto y evaluación del juego patológico. En J.L. Graña (Ed.) Conductas adictivas: teoría, evaluación y tratamiento. Madrid. Pirámide.

Echeburúa, E., Báez, C., Fernández-Montalvo, J. y Páez, D. (1994): Cuestionario de Juego Patológico de South Oaks (SOGS): validación española. Análisis y modificación de conducta, 20, 769-791.

Echeburúa, E., Báez, C. y Fernández, J. (1996): Comparative effectiveness of three therapeutic modalities in the psychological treatment of pathological gambling: long term outcome. Behavioural and cognitive psychotherapy, 24. $51-72$.

Feuerlein, W. (1976): Kurzfragebogen für Alcoholgefährdete (KFA): eine empirische analyse. Arch. psychiatr. nervenkr., 222, 139-152.

Hand, I., Fernández, F., Feiwdrich, B. (1995): Modelos de entendimiento del juego patológico: multimodal vs. adicción: implicaciones terapéuticas. Rev. psicol. salud, 1995, $\vee 7 ; n^{\circ} 2$; pags 19-32.

Legarda JJ; Babio R; y Abreu J M (1992): Prevalence estimates of pathological gambling in Seville (Spain). British journal of addictions, 87, 767-770.

Rodríguez-Martos, A. (1986): El diagnóstico del alcoholismo a través de cuestionarios. Barcelona: APAT, Fons informatiu, $n^{\circ} 2$.

Rosenthal, R. y Rugle, L. (1994): A psychodynamic approach to the treatment of pathological gambling: Achieving abstinence. Journal of gambling studies. Vol. 10 (I): $21-42$.

Spielberg, C.D., Gorsuch, R.L. y Lushene, R.E. (1970): Manual for the State/Trait anxiety inventory. Palo Alto C.A., Consulting Psychologists Press (versión española, TEA, 1982).

Volberg, R.A. y Steadman, H.J. (1988): Refining prevalence estimates of pathological gambling. American journal of psychiatry, 145. 502-505. 